\title{
Operationalizing a frailty index using routine blood and urine tests
}

This article was published in the following Dove Press journal:

Clinical Interventions in Aging

28 June 2017

Number of times this article has been viewed

\author{
Martin Ritt ${ }^{1,2}$ \\ Jakob Jäger ${ }^{2}$ \\ Julia Isabel Ritt ${ }^{2}$ \\ Cornel Christian Sieber ${ }^{1,3}$ \\ Karl-Günter Gaßmann ${ }^{1,2}$ \\ 'Institute for Biomedicine of \\ Ageing (IBA), Friedrich-Alexander \\ Universität Erlangen-Nürnberg (FAU) \\ Nuremberg, Germany; ${ }^{2}$ Department \\ of Internal Medicine III (Medicine of \\ Ageing), Geriatrics Center Erlangen, \\ Hospital of the Congregation of St \\ Francis Sisters of Vierzehnheiligen, \\ Erlangen, Germany; ${ }^{3}$ Department \\ of General Internal Medicine and \\ Geriatrics, Hospital of the Order of \\ St John of God, Regensburg, Germany
}

Correspondence: Martin Ritt

Department of Internal Medicine III (Medicine of Ageing), Geriatrics Center Erlangen, Hospital of the Congregation of St Francis Sisters of Vierzehnheiligen, 57 Rathsbergerstrasse, Erlangen 91054, Germany

Tel +49913 I 8223702

Fax +49 913। 8223703

Email martin.ritt@waldkrankenhaus.de
Background: Uncomplicated frailty instruments are desirable for use in a busy clinical setting. The aim of this study was to operationalize a frailty index (FI) from routine blood and urine tests, and to evaluate the properties of this FI compared to other frailty instruments.

Materials and methods: We conducted a secondary analysis of a prospective cohort study on 306 patients aged $\geq 65$ years hospitalized on geriatric wards. An FI comprising 22 routine blood parameters and one standard urine parameter (FI-Lab), a 50-item FI based on a comprehensive geriatric assessment (FI-CGA), a combined FI (FI-combined [items from the FI-Lab + others from the FI-CGA]), the Clinical Frailty Scale, rule-based frailty definition, and frailty phenotype were operationalized from data obtained during patients' hospital stays (ie, before discharge [baseline examination]). Follow-up data were obtained up to 1 year after the baseline examination.

Results: The mean FI-Lab score was $0.34 \pm 15$, with an upper limit of 0.74 . The FI-Lab was correlated with all the other frailty instruments (all $P<0.001$ ). The FI-Lab revealed an area under the receiver-operating characteristic curve (AUC) for 6-month and 1-year mortality of 0.765 (0.694-0.836) and $0.769(0.706-0.833)$, respectively (all $P<0.001)$. Each 0.01 increment in FI-Lab increased the risk (adjusted for age and sex) for 6-month and 1 -year mortality by $7.2 \%$ and $7.1 \%$, respectively (all adjusted $P<0.001$ ). When any of the other FIs (except the FI-combined) were also included in the models, each 0.01 increment in FI-Lab score was associated with an increase in the risk of 6-month and 1-year mortality by $4.1 \%-5.4 \%$ (all adjusted $P<0.001$ ).

Conclusion: The FI-Lab showed key characteristics of an FI. The FI-Lab can be applied as a single frailty measure or in combination with/in addition to other frailty instruments.

Keywords: older people, hospital, geriatric wards, frailty, risk stratification, mortality

\section{Introduction}

Worldwide, the older population is an ever-growing group ${ }^{1,2}$ and represents a large proportion of individuals treated and cared for in hospitals and/or outpatient settings in many countries. Some older people are active and fit, whereas others show complex health status with diverse, adverse medical conditions (eg, malnutrition, ${ }^{3,4}$ sarcopenia, ${ }^{5}$ and mobility impairment ${ }^{6}$ ), chronic diseases, and/or disability. These latter individuals may be frail or at least at risk of becoming frail. ${ }^{7-9}$ Frail older people show an increased vulnerability to stressors and have an increased risk in terms of adverse health outcomes, such as mortality. ${ }^{7,10-17}$ Some researchers consider frailty a medical syndrome (physical and/or cognitive) focusing particularly but not exclusively on nondisabled people..$^{10,17,18}$ Others view frailty as a broadly defined state of an individual. ${ }^{7-9}$ Nonetheless, there is overall agreement in the assumption that identification of frailty and/or its severity may aid improvements in management, decision making, and/or care planning for older people..$^{9,17,19-23}$ Frailty and its severity are considered to be excellent estimates 
of an older individual's biological age, which has been found to be a better indicator of an older person's mortality risk than his or her chronological age. ${ }^{24}$

Among the different instruments used to evaluate frailty, ${ }^{17,25-30}$ Fried et al's frailty phenotype ${ }^{10}$ and Rockwood et al's ${ }^{7}$ and Mitnitski et al's frailty index $(\mathrm{FI})^{11}$ are probably the best known. A limitation of the frailty phenotype is that it does not allow the grade of frailty severity to be differentiated. ${ }^{31}$ The FI represents a quantitative measure of the health status of an individual. ${ }^{32}$ It is the ratio of an individual's health deficits to the total number of health deficits evaluated. ${ }^{7,11}$ Accordingly, the more health deficits an individual demonstrates, the more frail he or she is considered to be. ${ }^{11}$ Health deficits used to construct an FI can embrace a whole range of health problems, including symptoms, signs, laboratory abnormalities, diseases, and disabilities. ${ }^{9}$ An FI can be operationalized on the basis of different data sets, ${ }^{8} \mathrm{eg}$, from data deriving from a comprehensive geriatric assessment (CGA). ${ }^{33-35}$ A further and major strength of the FI is that it allows a fine grading of the severity of frailty. ${ }^{11}$ The FI approach has repeatedly been found to be very powerful in identifying older people at high risk of mortality. ${ }^{7,34,36,37}$ A limitation of the FI is that the evaluation of individuals in relation to a larger list of clinical health deficits is time-consuming.

Recently, FIs based on routine blood tests (eg, white blood-cell count, hemoglobin, sodium, potassium, creatinine, and albumin) and standard physical measures (such as blood pressure and/or pulse) (FI-Lab) $)^{32,38,39}$ or at least in part on more specialized blood tests (eg, telomere length, DNA repair, and DNA repair:damage ratio) $(\mathrm{FI}-\mathrm{B})^{40}$ have been introduced and evaluated in community-dwelling and/or institutionalized persons. ${ }^{32,38-40}$ In these studies, ${ }^{32,38-40}$ the different FI-Lab measures ${ }^{32,38,39}$ and/or the FI-B ${ }^{40}$ were found to be powerful predictors of mortality. An FI based solely on routine laboratory parameters for a blood and/or urine sample might be an easy and feasible frailty instrument in a busy clinical setting, thereby overcoming the aforementioned limitations of a classical FI. Abnormal results in routine blood and/or urine tests might mirror preclinical health deficits and/or acute/chronic diseases. It is thus logical to suggest that different preclinical conditions and/or diseases might contribute additively or synergistically to a person's risk of dying or suffering from other adverse health outcomes. However, until now, no study has operationalized and evaluated an FI-Lab based solely on routine blood and/or urine tests. Moreover, no study has evaluated an FI-Lab or FI-B in a cohort of hospitalized patients.

Against this background, by utilizing a reanalysis of data from a recent prospective longitudinal analysis of a cohort of hospitalized patients on geriatric wards, ${ }^{12}$ we aimed to 1) operationalize an FI-Lab from routine laboratory parameters based on a blood and urine sample, 2) assess the relationship of the FI-Lab to other frailty instruments, and 3 ) investigate the predictive value of the FI-Lab in relation to 6-month and 1-year mortality compared to its individual items and other frailty instruments.

\section{Materials and methods Study design and study population}

This study was a secondary analysis of the database from a recent prospective, longitudinal study of 307 patients hospitalized on the geriatric wards of the Geriatrics Center Erlangen, Hospital of the Congregation of St Francis Sisters of Vierzehnheiligen, Erlangen, Germany. ${ }^{12}$ The inclusion criterion for this study was age 65 years or older. Exclusion criteria were the inability to give written informed consent or unavailability of a legal guardian to give written informed consent on behalf of the study participant. Blood and urine samples from the study participants were taken on admission, at various other time points during hospital stays, and before the patients were discharged. The blood and urine parameters assessed from samples taken from the patients before discharge were used for calculating the FI-Lab (baseline examination). In parallel, the patients were evaluated in terms of a broad spectrum of clinical characteristics (demographic data, functional impairments, diseases, Cumulative Illness Rating Scale - Geriatrics [CIRS-G], and Barthel Index, among other tools for a CGA) and different frailty instruments (a 50-item FI based on a comprehensive geriatric assessment [FI-CGA], the Clinical Frailty Scale, the rule-based frailty definition, and the frailty phenotype, among others) at the same time before discharge (baseline examination). So far, follow-up data have been obtained at 6 months and 1 year after the baseline examination. These data include information about the death of study participants during follow-up. Follow-up data were collected by using telephone interviews with patients, their physicians, specialists, relatives, or legal guardians. The study followed the principles of the Declaration of Helsinki and Good Clinical Practice. The study protocol was approved by the Ethics Committee of the University of Erlangen-Nuremberg. Written informed consent was obtained from each study participant or from his or her legal guardian.

\section{FI-Lab}

The FI-Lab as operationalized in this study was based on 22 routine parameters for a blood sample and one standard parameter for a spot urine sample. In selecting the individual parameters that construct the FI-Lab, care was taken that as a whole the FI-Lab captured information with respect to the 
functioning/health status of different bodily/organ systems. Consequently, the FI-Lab as used in this study includes hematological, inflammatory, coagulation, electrolyte, renal, liver, and thyroid parameters. The FI was constructed by coding each variable as either 0 or 1 . As such, 0 indicates that values were within the normal range or cutoffs (ie, no deficit present) and 1 that values were either above or below the normal range or cutoffs (ie, a deficit). The individual standard laboratory parameters that constitute the FI-Lab and their normal range or cutoff values are shown in Table 1. For calculation of the FI-Lab for any individual, the number of deficits was summed and divided by the number of potential deficits evaluated. Therefore, the FI-Lab for any individual resulted in a score ranging in magnitude from 0 to 1 . For example, a person with a deficit in five variables and no deficits in the other 18 variables of the 23-item FI-Lab would have an FI-Lab score of 0.217 ( 5 divided by 23). The FI-Lab was calculated only if more than $80 \%$ of the component variables were available for a given individual.

\section{FI-CGA}

The FI-CGA was composed of up to 50 items from Rockwood et al's 52-item FI-CGA. ${ }^{34}$ In contrast to the 52-item FI-CGA by Rockwood et a ${ }^{34}$ the 50 -item FI-CGA in the study presented here did not include the two items orthostatic hypotension and functional reach. In brief, the 50 different individual health deficits (items) used to construct the 50-item FI-CGA refer to nine different domains of a $\mathrm{CGA}^{33}$ (cognition, emotion, communication, mobility, balance, bladder function, bowel function, nutrition, and instrumental and basic activities of daily living), several diseases related to different organ systems, systolic and diastolic blood pressure levels, self-reported quality of life, and the number of medications. Each item of the FI-CGA was scored (according to the severity of the health deficit, with a maximum score of 1 per item) according to the criteria previously described in detail in the aforementioned work by Rockwood et al. ${ }^{34}$ The FI score was finally calculated as the score in each item divided by the total number of items evaluated, resulting in an FI-CGA score ranging in magnitude between 0 and 1.

\section{Fl-combined}

The FI-combined was operationalized based on the items of the aforementioned 23-item FI-Lab and the aforementioned 50-item FI-CGA. Therefore, the FI-combined was composed of 73 items. The FI-combined was calculated as the score in each item divided by the total number of items evaluated, resulting in an FI-combined score between 0 and 1.

Table I Standard laboratory parameters used to construct the frailty index based on routine blood and urine tests (FI-Lab)

\begin{tabular}{|c|c|c|c|c|c|}
\hline $\begin{array}{l}\text { Standard laboratory } \\
\text { parameter }\end{array}$ & Normal range/cutoff & $\begin{array}{l}\text { AUC }(95 \% \mathrm{CI}) \\
\text { for } 6 \text {-month } \\
\text { mortality }\end{array}$ & $P$-value & $\begin{array}{l}\text { AUC }(95 \% \mathrm{Cl}) \\
\text { for I-year } \\
\text { mortality }\end{array}$ & $P$-value \\
\hline \multicolumn{6}{|l|}{ Blood sample } \\
\hline White blood cells (number/ $\mu \mathrm{L}$ ) & $4,000-10,000$ & $0.592(0.498-0.686)$ & 0.045 & $0.589(0.505-0.673)$ & 0.03 \\
\hline Red blood cells (number/ $\mu \mathrm{L}$ ) & Men $4.5 \times 10^{6}-5.9 \times 10^{6}$, women $3.9 \times 10^{6}-5.1 \times 10^{6}$ & $0.53(0.44 I-0.6 I 8)$ & 0.519 & $0.546(0.466-0.625)$ & 0.269 \\
\hline Hemoglobin (g/dL) & Men 14-17.5, women 12.3-15.3 & $0.579(0.496-0.662)$ & 0.085 & $0.592(0.5 \mid 8-0.666)$ & 0.025 \\
\hline MCV (fL) & $80-96$ & $0.492(0.402-0.582)$ & 0.865 & $0.517(0.435-0.599)$ & 0.674 \\
\hline Hematocrit (\%) & Men 40-52, women 35-47 & $0.613(0.526-0.699)$ & 0.014 & $0.614(0.536-0.692)$ & 0.006 \\
\hline Platelets (number/ $\mu \mathrm{L}$ ) & $150,000-400,000$ & $0.476(0.379-0.572)$ & 0.6 & $0.47 \mathrm{I}(0.384-0.558)$ & 0.478 \\
\hline Quick value (\%) & $70-130$ & $0.562(0.469-0.654)$ & 0.179 & $0.54(0.458-0.622)$ & 0.332 \\
\hline PTT (seconds) & $25.1-36.5$ & $0.597(0.506-0.689)$ & 0.034 & $0.589(0.506-0.672)$ & 0.032 \\
\hline Sodium (mval/L) & $136-\mid 45$ & $0.562(0.47-0.655)$ & 0.176 & $0.566(0.483-0.649)$ & 0.109 \\
\hline Potassium (mg/dL) & $3.5-5.1$ & $0.56 \mid(0.466-0.656)$ & 0.183 & $0.535(0.452-0.618)$ & 0.39 \\
\hline Calcium (mval/L) & $4.3-5.2$ & $0.647(0.555-0.74)$ & 0.001 & $0.617(0.534-0.7)$ & 0.005 \\
\hline Protein, total (g/dL) & $6.4-8.7$ & $0.548(0.457-0.638)$ & 0.305 & $0.568(0.487-0.648)$ & 0.103 \\
\hline Urea $(\mathrm{mg} / \mathrm{dL})$ & $17-43$ & $0.572(0.483-0.661)$ & 0.116 & $0.564(0.483-0.644)$ & 0.122 \\
\hline Creatinine (mg/dL) & Men $0.7-1.2$, women $0.5-0.9$ & $0.504(0.415-0.594)$ & 0.922 & $0.517(0.436-0.597)$ & 0.687 \\
\hline Bilirubin $(\mathrm{mg} / \mathrm{dL})$ & $<1$ & $0.558(0.463-0.653)$ & 0.207 & $0.548(0.464-0.632)$ & 0.248 \\
\hline AST (SGOT, U/L) & Men $<35$, women $<3$ I & $0.57 \mid(0.477-0.665)$ & 0.123 & $0.573(0.489-0.657)$ & 0.076 \\
\hline ALT (SGPT, U/L) & Men $<45$, women $<34$ & $0.55(0.456-0.644)$ & 0.272 & $0.548(0.464-0.631)$ & 0.248 \\
\hline GGT (U/L) & Men $<55$, women $<33$ & $0.58(0.495-0.665)$ & 0.081 & $0.59(0.5 \mid 3-0.666)$ & 0.03 \\
\hline LDH (U/L) & Men $<248$, women $<247$ & $0.609(0.52-0.699)$ & 0.018 & $0.637(0.559-0.716)$ & 0.001 \\
\hline Albumin (g/dL) & $3.97-4.94$ & $0.612(0.53-0.693)$ & 0.015 & $0.606(0.53 \mathrm{I}-0.68 \mathrm{I})$ & 0.01 \\
\hline CRP (mg/dL) & $<0.5$ & $0.623(0.545-0.702)$ & 0.007 & $0.60 I(0.527-0.676)$ & 0.014 \\
\hline $\mathrm{TSH}(\mu \mathrm{U} / \mathrm{mL})$ & $0.27-4.2$ & $0.489(0.4-0.578)$ & 0.817 & $0.496(0.415-0.576)$ & 0.916 \\
\hline \multicolumn{6}{|l|}{ Spot urine } \\
\hline Protein, total (mg/dL) & $<15 \mathrm{mg} / \mathrm{dL}$ & $0.591(0.495-0.686)$ & $0.05 I$ & $0.576(0.492-0.661)$ & 0.066 \\
\hline
\end{tabular}

Abbreviations: AUC, area under the receiver-operating characteristic curve; $\mathrm{Cl}$, confidence interval; MCV, mean corpuscular volume; PTT, partial thromboplastin time; TSH, thyroid-stimulating hormone; AST, aspartate aminotransferase; SGOT, serum glutamic oxaloacetic transaminase; ALT, alanine aminotransferase; SGPT, serum glutamic Pyruvic transaminase; GGT, gamma-glutamyl transferase; LDH, lactate dehydrogenase; CRP, C-reactive proteine. 


\section{Clinical Frailty Scale}

The Clinical Frailty Scale consists of nine different categories, and grades individuals along a very fit (category 1 ) to terminally ill (category 9) continuum. ${ }^{17}$ The Clinical Frailty Scale is based on clinical judgment to interpret the results of history-taking and clinical examination. It takes physical activity, quality of disease control, functioning in instrumental and basic activities of daily living, history and severity of dementia, and the presence of terminal illnesses into account. ${ }^{17}$

\section{Rule-based frailty definition}

The four-level rule-based frailty definition was constructed as previously described in detail by Rockwood et al. ${ }^{41}$ It classifies persons in terms of frailty on a 4-point scale: level 0 (fit) to level 3 (frail), based on ability to walk, perform basic activities of daily living (eating, dressing, bathing, bed transfer), bowel function, bladder function, cognitive function, and the presence/absence of dementia. ${ }^{41}$

\section{Frailty phenotype}

The frailty phenotype is based on five phenotypic criteria (shrinking, ie, weight loss [unintentional]/loss of muscle mass, poor endurance/exhaustion, slowness, low physical activity, and muscle weakness). ${ }^{10}$ In the current study, the phenotypic criteria of the frailty phenotype were operationalized as described in detail elsewhere. ${ }^{36}$ Patients who revealed none of the phenotypic criteria were considered to be robust, those with one or two to be "prefrail", and those with three or more to be frail. ${ }^{36}$

\section{Statistics}

All statistical analyses were performed using SPSS software (IBM Corporation, Armonk, NY, USA). Results are expressed as mean \pm standard deviation, median (interquartile range), or percentages. Comparison between patients stratified according to different FI-Lab scores were performed using $\chi^{2}$ tests. Correlation analyses were performed using Spearman's $\rho$. Partial-correlation analyses were performed to evaluate the relationship between the FI-Lab and the other frailty instruments, taking potential confounders, ie, age and sex, into account. A correlation coefficient $r$ or partial $r$ of $0.9-1$ indicates "very high", 0.7-0.9 "high", 0.5-0.7 "moderate", 0.3-0.5 "low", and 0-0.3 "negligible" correlation. ${ }^{42}$ The various frailty instruments were analyzed as continuous variables, unless otherwise indicated. Receiver-operating characteristic curves were calculated to estimate area under the receiver-operating characteristic curve (AUCs) for the different items and frailty instruments in relation to mortality. AUC values $>0.9$ indicate "very good", $>0.8$ "good", and $>0.7$ "useful" predictive ability of the model.$^{43}$ An AUC value of 0.5 indicates that the predictive ability of the model is not better than chance. ${ }^{43}$ Comparisons among the AUCs were performed using the method of Hanley and McNeil. ${ }^{44}$ Kaplan-Meier estimates were used to determine whether different patients with different FI-Lab scores differed in their ability to predict 6-month and 1-year mortality. The $P$-value reported for the difference with reference to mortality among the patient groups with different FI-Lab scores was based on the log-rank test. Cox proportional hazard models were used to estimate the probability of survival, in which FI-Lab, FI-CGA, and FI-combined values were converted to $0-100$ integers by rounding them after multiplying them by 100 , giving equal percentage increments for modeling. Hazard ratios (HRs) of the FI-Lab and the other frailty instruments were adjusted for age and sex, among other variables, and were considered both separately and together. The level of statistical significance was set a priori at $P<0.050$.

\section{Results}

For 306 of the 307 patients, more than $80 \%$ of the laboratory parameters that comprise the FI-Lab were available. The patient with an availability of less than $80 \%$ of the laboratory parameters comprising the FI-Lab was excluded from the analysis. This person was an 87 -year-old male who had a body mass index (BMI) of $28 \mathrm{~kg} / \mathrm{m}^{2}$, a Mini-Mental State Examination (MMSE) score of 70 points, a Barthel Index score of 70 points, and a CIRS-G score of 22 points before discharge (at the baseline examination). Follow-up data at 6-month follow-up were available from all the remaining 306 study participants; 47 of these (15.4\%) had died by that point. Follow-up data at 1 year were not available for two patients of the aforementioned 306 study participants. The two patients for whom no follow-up data were available at 1 year were $89 \pm 4.2$ years old, one woman and one man, had a BMI of $30.7 \pm 8.8 \mathrm{~kg} / \mathrm{m}^{2}$, an MMSE score of $27.5 \pm 0.7$ points, a Barthel Index score of $70 \pm 7.1$, CIRS-G score of $10 \pm 2.8$ points, and an FI-Lab score of $0.28 \pm 0.2$ before discharge (at the baseline examination). Of the remaining 304 patients for whom follow-up data were available at 1 year of follow-up, 62 patients (20.4\%) had died by that point.

The 306 patients for whom more than $80 \%$ of the laboratory parameters that constitute the FI-Lab were available were $82.9 \pm 6.4$ years old, $67.6 \%$ female, $163 \pm 9.7 \mathrm{~cm}$ tall, weighed $73.2 \pm 16 \mathrm{~kg}$, with BMI scores of $27.5 \pm 5.6 \mathrm{~kg} / \mathrm{m}^{2}$; 
$25.8 \%$ had weight loss $>4.5 \mathrm{~kg}$ in the previous year, MMSE scores were $25.5 \pm 4.6$ points, Geriatric Depression Scale scores were $3.88 \pm 2.7$ points, $63.7 \%$ had timed "up and go" (TUG) results $>19$ seconds or were unable to perform the TUG, Barthel Index scores were $70.1 \pm 21$ points, CIRS-G scores were $17.4 \pm 5.6$ points; and $47.4 \%$ had a history of heart failure, $13.7 \%$ myocardial infarction, $17.6 \%$ peripheral vascular disease, $20.3 \%$ stroke, $14.7 \%$ cancer, $37.9 \%$ diabetes mellitus, $16.7 \%$ lung disease, $64.7 \%$ kidney disease, $23.5 \%$ urinary incontinence or catheterized, $31.7 \%$ constipation, $96.1 \%$ received more than five medications, and $16 \%$ were institutionalized. The clinical characteristics and scores of the different frailty instruments of these aforementioned patients stratified according to the different FI-Lab scores are given in Table 2. Patients with higher FI-Lab scores included a greater percentage of male patients, patients with weight loss $>4.5 \mathrm{~kg}$ in the last year, those with lower MMSE scores, a greater percentage of patients with a TUG $>19$ seconds or unable to perform the TUG, a greater comorbidity burden as assessed by the CIRS-G, a greater percentage of patients with heart failure, peripheral vascular disease, kidney disease, urinary incontinence or catheterized, constipation, more than five medications, FI-CGA scores, FI-combined scores, Clinical Frailty Scale categories, rule-based frailty-definition level, frail state (three or more phenotypic components) according to the frailty phenotype, and higher 6-month and 1-year mortality compared to patients with lower FI-Lab scores.

FI-Lab scores showed normal distribution. The mean FI-Lab score was $0.34 \pm 0.15$, and the median $0.34(0.22-0.43)$. The minimum FI-Lab score observed was 0 , while the maximum was 0.74 . The first, fifth, 95th, and 99th percentiles of the FI-Lab were $0.04,0.09,0.57$, and 0.7 , respectively. Univariate correlation analysis revealed relationships between the FI-Lab and the FI-CGA, FI-combined, Clinical Frailty Scale, rule-based frailty definition, and frailty phenotype $(r=0.497[P<0.001], r=0.739[P<0.001], r=0.483$ $[P<0.001], r=0.37[P<0.001]$, and $r=0.4[P<0.001]$, respectively). Relationships between the FI-Lab and the FI-CGA, FI-combined, Clinical Frailty Scale, rule-based frailty definition, and frailty phenotype were independent of age and sex (partial $r=0.478$ [adjusted $P<0.001$ ], partial $r=0.734$ [adjusted $P<0.001$ ], partial $r=0.458$ [adjusted $P<0.001$ ], partial $r=0.355$ [adjusted $P<0.001$ ], and partial $r=0.376$ [adjusted $P<0.001$ ], respectively).

The FI-Lab revealed at least useful discriminative accuracy (ie, AUC >0.7) for 6-month and 1-year mortality (Table 3). Several individual items of the FI-Lab, ie, white blood cells, hemoglobin, hematocrit, partial thromboplastin time, calcium, GGT, LDH, albumin, and CRP were able to discriminate between patients who had died and those who had not during the 6-month and/or 1-year follow-up periods (Table 1). However, when considered individually, the items of the FI-Lab had rather poor discriminative accuracy (Table 1). The discriminative accuracy of the FI-Lab for 6-month and 1-year mortality was greater than any individual item of the FI-Lab (all $P<0.05$ ).

In addition to the FI-Lab, all the other frailty instruments evaluated in this study were also able to discriminate between patients who had died and those who were alive at 6-month and 1-year follow-ups (Table 3). The discriminative accuracy of the FI-Lab for 6-month mortality was inferior compared to the FI-CGA, FI-combined, and the Clinical Frailty Scale (all $P<0.05$, Table 3 ) and similar compared to the rule-based frailty definition and the frailty phenotype (both $P \geq 0.05$, Table 3 ). The discriminative accuracy of the FI-Lab for 1-year mortality was inferior to the FI-combined and the Clinical Frailty Scale (all $P<0.05$, Table 3 ), similar to the FI-CGA and the frailty phenotype (both $P \geq 0.05$, Table 3), and superior to the rule-based frailty definition $(P<0.05$, Table 3$)$.

Unadjusted and adjusted HRs for each increment in category/level or 0.01 increments in score of the different frailty instruments are given in Table 4 . Each $1 \%$ increase in FI-Lab scores (each 0.01 increment) increased HRs for 6-month and 1 -year mortality by $7.2 \%$ and $7.1 \%$, respectively (Table 4). In an age- and sex-adjusted model, each 0.01 increment in FI-Lab score increased HRs for 6-month and 1-year mortality by $7.2 \%$ and $7.1 \%$, respectively (Table 4 ). When the FI-CGA, Clinical Frailty Scale, rule-based frailty definition, or frailty phenotype were also entered into the models, each 0.01 increment in FI-Lab score still showed HRs for 6-month and 1-year mortality of 4.1\%-5.4\% (Table 5).

\section{Discussion}

We operationalized an FI-Lab from 22 routine laboratory parameters based on a blood sample and one standard parameter based on a urine sample in 306 hospitalized patients on geriatric wards. Howlett et $\mathrm{al}^{32}$ and Rockwood et $\mathrm{al}^{38}$ evaluated a 23-item FI-Lab, which was based on 21 routine blood tests plus standard physical measures (ie, systolic and diastolic blood pressure), ${ }^{32,38}$ in community-dwelling older people and/or institutionalized individuals. Similarly, Blodgett et $\mathrm{al}^{39}$ evaluated a 23-item FI-Lab, which was constructed by routine blood tests plus standard physical measures (ie, blood pressure and pulse), in communitydwelling older men. Mitnitski et $\mathrm{al}^{40}$ evaluated an FI-B based 


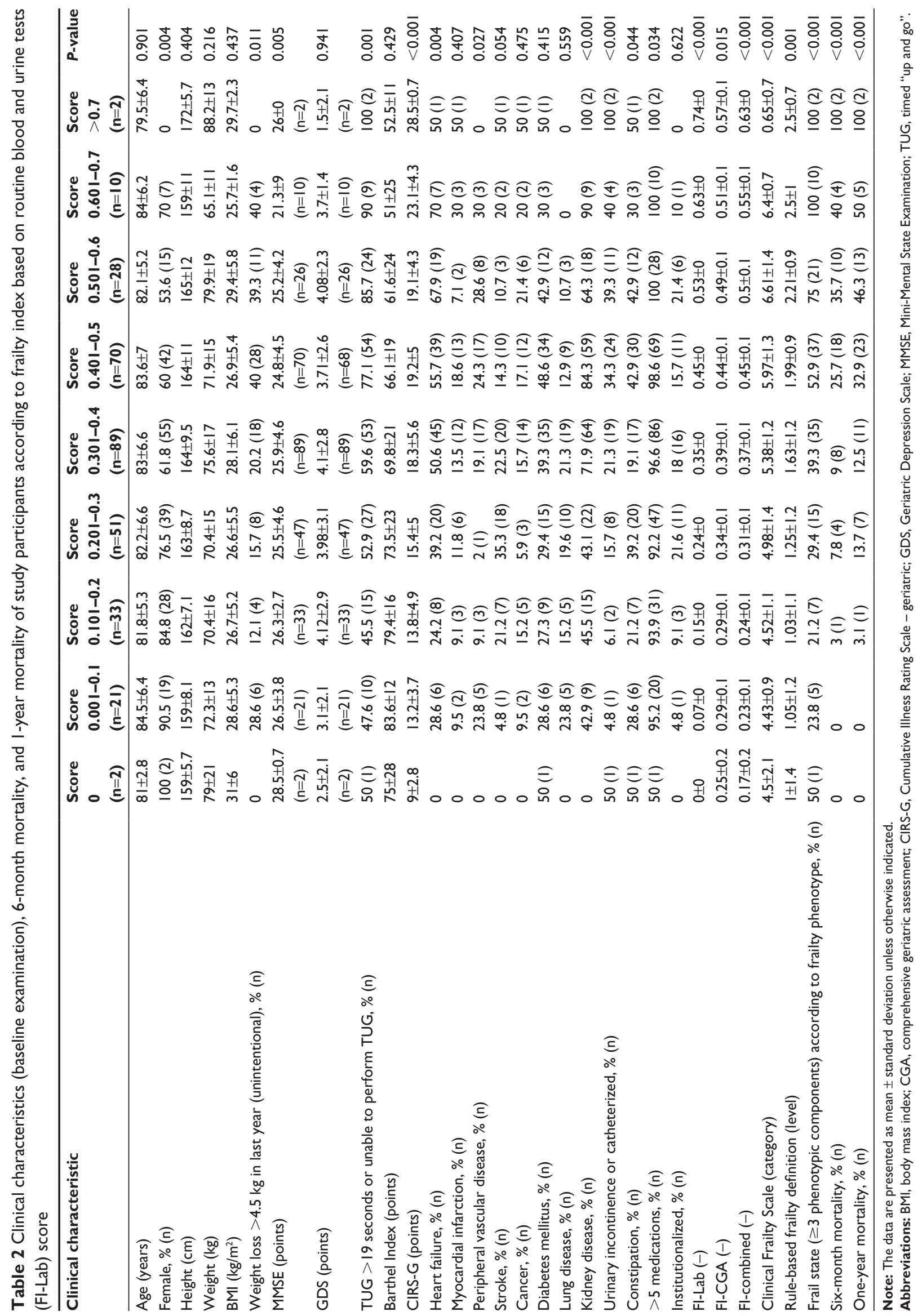


Table 3 Ability of different frailty instruments and comparison of frailty index based on routine blood and urine tests (FI-Lab) with other frailty instruments to predict 6-month and I-year mortality

\begin{tabular}{|c|c|c|c|c|c|c|}
\hline Frailty instrument & $\begin{array}{l}\text { AUC }(95 \% \mathrm{Cl}) \\
\text { for 6-month } \\
\text { mortality }(n=306)\end{array}$ & $P$-value & $\begin{array}{l}\text { P-value, } \\
\text { AUC I vs } \\
\text { AUCs 2-7 }\end{array}$ & $\begin{array}{l}\text { AUC }(95 \% \mathrm{CI}) \\
\text { for I-year } \\
\text { mortality }(n=304)\end{array}$ & $P$-value & $\begin{array}{l}\text { P-value, } \\
\text { AUC I vs } \\
\text { AUCs 2-7 }\end{array}$ \\
\hline FI-Lab & $0.765(0.694-0.836)$ & $<0.001$ & - & $0.769(0.706-0.833)$ & $<0.001$ & - \\
\hline $\mathrm{FI}-\mathrm{CGA}$ & $0.834(0.767-0.90 I)$ & $<0.001$ & 0.030 & $0.806(0.744-0.867)$ & $<0.001$ & 0.139 \\
\hline Fl-combined & $0.853(0.792-0.915)$ & $<0.001$ & $<0.001$ & $0.832(0.776-0.888)$ & $<0.001$ & 0.004 \\
\hline Clinical Frailty Scale & $0.867(0.807-0.926)$ & $<0.001$ & 0.002 & $0.852(0.8-0.904)$ & $<0.001$ & 0.005 \\
\hline Rule-based frailty definition & $0.716(0.642-0.79)$ & $<0.00$ I & 0.126 & $0.703(0.638-0.769)$ & $<0.001$ & 0.040 \\
\hline Frailty phenotype & $0.754(0.687-0.82)$ & $<0.001$ & 0.391 & 0.724 (0.659-0.79) & $<0.001$ & 0.118 \\
\hline
\end{tabular}

Abbreviations: AUC, area under the receiver-operating characteristic curve; CGA, comprehensive geriatric assessment; $\mathrm{Cl}$, confidence interval.

on 40 biomarkers that included at least in part more special blood tests (eg, cytomegalovirus serology [IgG], senescent memory CD4 T cells, telomere length, DNA repair, DNA damage:repair ratio, and mitochondrial DNA haplogroup) in a population-based cohort of individuals aged 85 years or older. As operationalized in our current study, the FI-Lab differs from the aforementioned FI-Labs ${ }^{32,38,39}$ and the aforementioned FI-B by Mitnitski et al ${ }^{40}$ In contrast to the different FI-Labs of the aforementioned authors, ${ }^{32,38,39}$ no standard physical measures (such as blood pressure and/or pulse) were included to operationalize the FI-Lab in the study presented here. In addition, at least some routine blood tests used to construct FI-Lab differed between the FI-Labs of the aforementioned authors ${ }^{32,38,39}$ and the FI-Lab in our current study. In contrast to the FI-B of Mitnitski et al, ${ }^{40}$ only routine laboratory blood parameters and no more special blood tests were used to operationalize the FI-Lab presented here. One strength of the FI-Lab, as operationalized in our current study, is that it can be analyzed from a single laboratory report from a general laboratory without the need for consideration of additional parameters, such as standard physical tests or more specialized blood tests.

The FI-Lab evaluated in our study showed valuable discriminatory accuracy, as indicated by an AUC $>0.70$ for 6-month and 1-year mortality. Clearly, abnormalities in routine blood and/or urine parameters might reflect subclinical organ changes, adverse medical conditions, and/or acute or chronic diseases that potentially impact on an older person's mortality risk. In addition, an interaction between chronic diseases and functional impairments that impact on the mortality risk of older persons has been previously reported by other authors. ${ }^{45-47}$ Therefore, several of the aforementioned conditions might have driven the predictive power of the FI-Lab in our current study. Patients with higher FI-Lab scores showed a greater overall comorbidity burden, as indicated by higher CIRS-G score and a greater percentage of patients with heart failure, peripheral vascular disease, kidney disease, urinary incontinence or catheterized, constipation,

Table 4 HRs and adjusted (for age and sex) HRs for each increment in category or score of 0.0 I of the different frailty instruments in relation to 6-month and I-year mortality

\begin{tabular}{|c|c|c|c|c|}
\hline Frailty instrument & HR (95\% Cl) & $P$-value & Adjusted HR (95\% Cl) & $P$-value \\
\hline \multicolumn{5}{|l|}{ Six-month mortality } \\
\hline Fl-Lab (per each 0.01 increment in score) & $1.072(1.048-1.096)$ & $<0.001$ & $1.072(1.048-1.097)$ & $<0.001$ \\
\hline FI-CGA (per each 0.01 increment in score) & $1.105(1.076-1.135)$ & $<0.00$ I & $1.102(1.073-1.132)$ & $<0.001$ \\
\hline Fl-combined (per each 0.01 increment in score) & $1.134(1.099-1.171)$ & $<0.001$ & $1.133(1.098-1.17)$ & $<0.001$ \\
\hline Clinical Frailty Scale (per each increment in category [I-9]) & $2.614(2.132-3.206)$ & $<0.001$ & $2.545(2.055-3.15)$ & $<0.001$ \\
\hline Rule-based frailty definition (per each increment in level [0-3]) & $2.333(1.613-3.373)$ & $<0.001$ & $2.259(1.554-3.285)$ & $<0.001$ \\
\hline Frailty phenotype (per increase in category [robust/prefrail/frail]) & $6.063(2.983-12.325)$ & $<0.001$ & $5.618(2.754-11.46)$ & $<0.001$ \\
\hline \multicolumn{5}{|l|}{ One-year mortality } \\
\hline FI-Lab (per each 0.01 increment in score) & $1.07 \mid(1.05-1.092)$ & $<0.001$ & $1.07 \mid(1.05-1.093)$ & $<0.001$ \\
\hline 50-item FI-CGA (per each 0.01 increment in score) & $1.089(1.066-1.113)$ & $<0.001$ & $1.087(1.064-1.112)$ & $<0.001$ \\
\hline Fl-combined (per each 0.01 increment in score) & $1.119(1.09-1.148)$ & $<0.001$ & $1.118(1.088-1.148)$ & $<0.001$ \\
\hline Clinical Frailty Scale (per each increment in category [I-9]) & $2.569(2.128-3.1)$ & $<0.001$ & $2.515(2.069-3.059)$ & $<0.001$ \\
\hline Rule-based frailty definition (per each increment in level [0-3]) & $2.189(1.611-2.975)$ & $<0.001$ & $2.142(1.567-2.929)$ & $<0.001$ \\
\hline Frailty phenotype (per increase in category [robust/prefrail/frail]) & $3.977(2.389-6.622)$ & $<0.001$ & $3.744(2.24 I-6.257)$ & $<0.001$ \\
\hline
\end{tabular}

Abbreviations: CGA, comprehensive geriatric assessment; Cl, confidence interval; FI-Lab, frailty index (based on routine blood and urine tests); HR, hazard ratio. 
Table 5 Adjusted HRs for age, sex, and each increment in category/level or score of 0.01 of the frailty index based on Fl-Lab, $\mathrm{FI}-\mathrm{CGA}$, rule-based frailty definition, and frailty phenotype in relation to 6-month and I-year mortality

\begin{tabular}{|c|c|c|}
\hline Variable & Adjusted HR (95\% Cl) & $P$-value \\
\hline \multicolumn{3}{|l|}{ Six-month mortality } \\
\hline Age & $1.026(0.974-1.081)$ & 0.329 \\
\hline Sex & $0.747(0.4 \mid 8-1.333)$ & 0.324 \\
\hline Fl-Lab (per each 0.01 increment in score) & $1.046(1.02-1.073)$ & 0.001 \\
\hline Clinical Frailty Scale (per each increment in category [1-9]) & $2.287(1.83-2.858)$ & $<0.001$ \\
\hline Age & $1.046(0.995-1.099)$ & 0.077 \\
\hline Sex & $0.711(0.399-1.269)$ & 0.249 \\
\hline FI-Lab (per each $0.0 \mathrm{I}$ increment in score) & $1.041(1.017-1.066)$ & 0.001 \\
\hline FI-CGA (per each 0.01 increment in score) & $1.087(1.056-1.119)$ & $<0.001$ \\
\hline Age & $1.064(1.01-1.12)$ & 0.019 \\
\hline Sex & $0.693(0.387-\mid .24 I)$ & 0.217 \\
\hline Fl-Lab (per each $0.0 \mathrm{I}$ increment in score) & $1.054(1.028-1.08)$ & $<0.001$ \\
\hline Rule-based frailty definition (per each increment in level [0-3]) & $3.562(1.915-6.627)$ & $<0.001$ \\
\hline Age & $1.052(1.001-1.107)$ & 0.047 \\
\hline Sex & $0.716(0.402-1.275)$ & 0.257 \\
\hline FI-Lab (per each $0.0 \mathrm{I}$ increment in score) & $1.051(1.026-1.076)$ & $<0.001$ \\
\hline Frailty phenotype (per increase in category [robust/prefrail/frail]) & $3.869(1.852-8.085)$ & $<0.001$ \\
\hline \multicolumn{3}{|l|}{ One-year mortality } \\
\hline Age & $1.027(0.974-1.082)$ & 0.322 \\
\hline Sex & $0.745(0.417-1.33)$ & 0.319 \\
\hline FI-Lab (per each $0.0 \mathrm{I}$ increment in score) & $1.046(1.02-1.073)$ & 0.001 \\
\hline Clinical Frailty Scale (per each increment in category [I-9]) & $2.282(1.826-2.852)$ & $<0.001$ \\
\hline Age & $1.046(0.995-1.099)$ & 0.076 \\
\hline Sex & $0.711(0.398-1.268)$ & 0.248 \\
\hline FI-Lab (per each $0.0 \mathrm{I}$ increment in score) & $1.041(1.017-1.066)$ & 0.001 \\
\hline FI-CGA (per each 0.01 increment in score) & $1.087(1.056-1.119)$ & $<0.001$ \\
\hline Age & $1.065(1.011-1.121)$ & 0.018 \\
\hline Sex & $0.69(0.385-1.236)$ & 0.212 \\
\hline Fl-Lab (per each 0.01 increment in score) & $1.053(1.027-1.079)$ & $<0.001$ \\
\hline Rule-based frailty definition (per each increment in level [0-3]) & $3.558(1.913-6.616)$ & $<0.001$ \\
\hline Age & $1.053(1.001-1.108)$ & $<0.045$ \\
\hline Sex & $0.7 \mid 3(0.4-1.27 \mid)$ & 0.251 \\
\hline FI-Lab (per each $0.0 \mathrm{I}$ increment in score) & $1.05(1.026-1.075)$ & $<0.001$ \\
\hline Frailty phenotype (per increase in category [robust/prefrail/frail]) & $3.828(1.835-7.985)$ & $<0.001$ \\
\hline
\end{tabular}

Abbreviations: CGA, comprehensive geriatric assessment; Cl, confidence interval; FI-Lab, frailty index (based on routine blood and urine tests); HR, hazard ratio.

polypharmacy (more than five medications), lower cognitive function (lower MMSE score), mobility impairment (TUG $>19$ seconds or unable to perform the TUG), and weight loss $>4.5 \mathrm{~kg}$ in the last year compared to patients with lower FI-Lab scores. In accordance with the findings of this study, in studies by other authors, the comorbidity burden as assessed by the CIRS-G, ${ }^{48,49}$ heart failure, ${ }^{50}$ peripheral vascular disease, ${ }^{51}$ kidney disease, ${ }^{52}$ impairment in bladder function, ${ }^{53,54}$ constipation, ${ }^{55}$ polypharmacy, ${ }^{56}$ impairment in cognition, ${ }^{57,58}$ mobility impairment, ${ }^{59,60}$ and weight $\operatorname{loss}^{61}$ were found to be associated with a greater mortality risk in older people. In addition, in the study presented here, patients with higher FI-Lab scores included more male than female patients compared to patients with lower FI-Lab scores. In previous studies on older individuals, ${ }^{55,62}$ a greater risk for mortality in males than females was found. However, it is worth noting that in our study, the predictive power of the FI-Lab was found to be independent of the sex of the patients.

The FI-Lab evaluated here showed a fine grading of the patients' 6-month and 1-year mortality risk independently of age and sex. In line with our findings, such a fine grading of patient-mortality risk has also been found previously with an FI. ${ }^{7,63}$ Of interest, the risk for 6-month and 1-year mortality captured by the FI-Lab, as indicated by the HR, was independent of that of the Clinical Frailty Scale, FI-CGA, rule-based frailty definition, or the frailty phenotype. Consequently, the FI-Lab might be of additional value in terms of any of the other aforementioned frailty instruments when applied 
to evaluate a person's mortality risk. Concordant with this, Rockwood et $\mathrm{al}^{38}$ detected prognostic significance in relation to 6-year mortality with a 23-item FI-Lab based on standard parameters of a blood sample plus blood pressure levels that was independent of age, sex, and a 58-item clinical FI in a cohort of institutionalized older persons.

The FI-Lab differed at least in part in its accuracy to predict 6-month and/or 1-year mortality compared to other frailty instruments that were evaluated in this study. The FI-Lab showed superior discriminative accuracy for mortality to the rule-based frailty definition (for 1-year mortality), similar discriminative accuracy for mortality to the FI-CGA (for 1-year mortality), the frailty phenotype (for 6-month and 1-year mortality), and the rule-based frailty definition (for 6-month mortality), and inferior discriminative accuracy for mortality to the FI-CGA (for 6-month mortality), FI-combined (for 6-month and 1-year mortality) and Clinical Frailty Scale (for 6-month and 1-year mortality). The inferior ability of the FI-Lab to predict 6-month and/or 1-year mortality compared to some of the aforementioned frailty instruments might be due to the fact that routine blood and urine samples in general include only a smaller list of basic laboratory parameters. Consideration of a larger list of blood and urine parameters, which together capture more information with respect to the functioning/health status of different bodily/ organ systems, would probably improve estimation of mortality by applying an FI-Lab. This issue should be addressed in future studies. Of note, Howlett et al ${ }^{32}$ reported similar discriminative accuracy for 6-year mortality for their 23-item FI-Lab constructed by using standard laboratory parameters of a blood sample plus blood pressure levels and a 38-item FI based on clinical parameters in older community-dwelling or institutionalized people. In Mitnitski et al, ${ }^{40}$ the discriminative accuracy of a 40-item FI-B constructed by using standard biomarkers and more special laboratory parameters based on a blood sample for 7-year mortality did not differ (in terms of statistical significance) compared to a 40-item FI based on clinical parameters and the frailty phenotype in a populationbased sample of individuals aged 85 years or older. However, it should be taken into account that the follow-up periods of these studies were substantially longer compared to the follow-up periods of our current study.

The discriminative accuracy for 6-month and 1-year mortality of the FI-Lab evaluated in this study was superior to the discriminative accuracy of its individual items. The individual items of the FI-Lab that showed discriminative accuracy for 6-month and/or 1-year mortality included white blood cells, hemoglobin, hematocrit, partial thromboplastin time, calcium, GGT, LDH, albumin, and CRP. In accordance with the findings of this study, individual abnormal standard blood parameters have previously been found to predict mortality. Mitnitski et $\mathrm{a}^{40}$ reported a predictive value for 7-year mortality of abnormal white blood-cell count, hemoglobin, albumin, and highly sensitive CRP in a community-based cohort of people aged 85 years or older. With respect to abnormal albumin levels, hypoalbuminemia is a well-established prognostic marker for increased mortality risk in older people. ${ }^{64}$ Koehler et $\mathrm{al}^{65}$ detected an association between abnormal GGT values and mortality in older people. Wulaningsih et $\mathrm{al}^{66}$ and Liu et $\mathrm{l}^{67}$ found that increased serum LDH values predicted mortality.

The FI-Lab correlated with the other frailty instruments evaluated in this study independently of age and sex. Therefore, a substantial proportion of the patients in our cohort with greater frailty severity according to the FI-Lab also revealed greater frailty severity or at least a frail state according to the other frailty instruments and vice versa. In line, Rockwood et al $^{38}$ detected a relationship between a 23-item FI-Lab constructed by using standard parameters of a blood sample plus blood pressure levels and a 58-item FI based on clinical parameters in a cohort of institutionalized older people. Similarly, Mitnitski et $\mathrm{al}^{40}$ observed a relationship between a 40-item FI-B made up of multiple biomarkers, including several that went beyond standard laboratory tests from a blood sample and a 40-item FI based on clinical parameters in a population-based sample of people 85 years or older.

The frequency distribution of the FI-Lab showed a maximum FI-Lab score of 0.74 . This is in accordance with an upper limit to frailty for an FI score of approximately 0.7. Such an upper limit to frailty for an FI score of approximately 0.7 has frequently been detected in different study populations where an FI was applied. . $4,36,68,69$

In 2013, a consensus document ${ }^{17}$ was published that focused on a specific construct of frailty, ie, physical frailty. It was noted and emphasized that a broader construct of frailty and the construct of physical frailty should be conceived. ${ }^{17}$ In this consensus document ${ }^{17}$ work by Rockwood et $\mathrm{al}^{7}$ has been referenced as an example for the broader construct of frailty. The theoretical background to the frailty approach by Rockwood and Mitnitski is described in detail elsewhere. ${ }^{8,9}$ In brief, Rockwood and Mitnitski focused on the overall health state of a person as a measure of frailty. ${ }^{8}$ This frailty approach does not exclude per se the mention and/or consideration of disability. ${ }^{8}$ This is due to the notion that a great proportion of people who are frequently frail also show at least some degree of disability. ${ }^{8}$ In addition, the presence of 
a disability is frequently associated with increased mortality risk in older persons. ${ }^{8}$ Considering disability among other parameters thus seems appropriate in light of graded frailty severity and mortality risk in older people. ${ }^{8}$ In contrast to the broader construct of frailty, the construct of physical frailty targets "predisabled" people in particular (but not exclusively). ${ }^{17}$ Moreover, and of note, disability is being considered an outcome of the construct of physical frailty. ${ }^{17}$ Patients hospitalized on geriatric wards frequently show at least some disabilities. ${ }^{70,71}$ This was also the case in a large proportion of the study participants in our current study. In this light, we consider the broader construct of frailty in accordance with the approach by Rockwood et $\mathrm{al}^{7-9}$ as the very definition of frailty in the study presented here and as the conceptual frame in which this work is being developed.

This study has some major strengths. To the best of our knowledge, this is the first study to have evaluated an FI-Lab based solely on routine laboratory parameters of a blood and/or urine sample. In addition, this is the first study to evaluate an FI-Lab in a cohort of hospitalized patients. Moreover, we analyzed the predictive power of the FI-Lab for 6-month and 1-year mortality compared to its individual items and other frailty instruments. The prognostic power of a frailty instrument in relation to mortality might vary between different follow-up periods, in particular where the frailty instrument is based on items that reflect not only clinical but also subclinical deficits, such as the FI-Lab in our study.

This study has some limitations. The FI-Lab and the other frailty instruments were operationalized based on parameters that were evaluated at the end of the hospital stays of the study participants, ie, before discharge. At the end of the study participants' hospital stays, the acute diseases or exacerbations of chronic diseases that resulted in admission to hospital had been treated and controlled. The analysis of the patients in relation to frailty after treatment and control of acute diseases and exacerbations of chronic diseases might enable a more objective evaluation of the patients in relation to frailty and its severity according to the aforementioned frailty instruments than applying these tools in situations in which patients suffer from acute diseases or exacerbations of chronic diseases. Acute diseases or exacerbations of chronic disease might impact on laboratory, clinical, and functional parameters. Accordingly, it might be misleading to extrapolate the findings of our current study to patients in a situation in which they suffer from acute diseases and/or exacerbations of chronic diseases at the beginning of their hospital stays at geriatric wards. In relation to the frailty phenotype, in the study presented here, as well as other studies, ${ }^{72,73}$ the operationalization of the phenotypic components used differed slightly from the original operationalization by Fried et al. ${ }^{10}$ This might have reduced the ability of the frailty phenotype in our study to predict 6-month and 1 -year mortality. All the study participants were treated and cared for on geriatric wards. Extrapolation of the findings of the study presented here to other patients groups or clinical settings might thus be misleading.

\section{Conclusion}

In our study of 306 patients hospitalized on geriatric wards, an FI-Lab based solely on 23 routine laboratory parameters from blood and urine samples was associated with useful discriminative accuracy (AUC >0.7) and graded risk of 6-month and 1-year mortality. The predictive power of the FI-Lab for 6-month and 1-year mortality was independent of age, sex, and other frailty instruments. Frailty severity evaluated by FI-Lab was related to frailty status or severity assessed by other frailty instruments. The upper limit of the FI-Lab score in this study is in line with an upper limit of an FI of approximately 0.7. Accordingly, the FILab showed key characteristics of an FI. The FI-Lab, used as a single tool or in combination with/in addition to other frailty measures, emerged as a valuable frailty instrument for the estimation of mortality risk of patients hospitalized on geriatric wards.

\section{Acknowledgment}

This work was supported by a grant from the Robert Bosch Foundation (scholarship based on the Research and Study Program on Geriatrics) to MR.

\section{Disclosure}

The authors report no conflicts of interest in this work.

\section{References}

1. Crimmins EM. Lifespan and healthspan: past, present, and promise. Gerontologist. 2015;55:901-911.

2. Jagger C, Matthews F, Wohland P, et al. A comparison of health expectancies over two decades in England: results of the Cognitive Function and Ageing Study I and II. Lancet. 2016;387:779-786.

3. Christner S, Ritt M, Volkert D, Wirth R, Sieber CC, Gaßmann KG. Evaluation of the nutritional status of older hospitalised geriatric patients: a comparative analysis of a Mini Nutritional Assessment (MNA) version and the Nutritional Risk Screening (NRS 2002). J Hum Nutr Diet. 2016; 29:704-713.

4. Eschbach D, Kirchbichler T, Wiesmann T, et al. Nutritional intervention in cognitively impaired geriatric trauma patents: a feasibility study. Clin Interv Aging. 2016;11:1239-1246.

5. Kemmler W, Teschler M, Goisser S, et al. Prevalence of sarcopenia in Germany and the corresponding effect of osteoarthritis in females 70 years and older living in the community: results of the FORMoSA study. Clin Interv Aging. 2015;10:1565-1573. 
6. RittM, Schülein S, Lubrich H, Bollheimer LC, SieberCC, Gaßmann KG. High-technology based gait assessment in frail people: association between spatio-temporal and three-dimensional gait characteristics with frailty status across four different frailty measures J Nutr Health Aging. 2017;21:346-353.

7. Rockwood K, Song X, MacKnight C, et al. A global clinical measure of fitness and frailty in elderly people. CMAJ. 2005;5:489-495.

8. Rockwood K, Mitnitski A. How might deficit accumulation give rise to frailty? J Frailty Aging. 2012;1:8-12.

9. Rockwood K, Mitnitski A. Frailty defined by deficit accumulation and geriatric medicine defined by frailty. Clin Geriatr Med. 2011;27:17-26.

10. Fried LP, Tangen CM, Walston J, et al. Frailty in older adults: evidence for a phenotype. J Gerontol A Biol Sci Med Sci. 2001;56: M146-M156.

11. Mitnitski A, Mogilner AJ, Rockwood K. Accumulation of deficits as a proxy measure of ageing. ScientificWorldJournal. 2001;1:323-336.

12. Ritt M, Bollheimer LC, Sieber CC, Gaßmann KG. Prediction of one-year mortality by five different frailty instruments: a comparative study in hospitalized geriatric patients. Arch Gerontol Geriatr. 2016;66:66-72.

13. Liuz Z, Wang Q, Zhi T, et al. Frailty index and its relation to falls and overnight hospitalizations in elderly Chinese people: a population-based study. J Nutr Health Aging. 2016;20:561-568.

14. Romero-Ortuno R, Wallis SJ, Biram RW, Keevil V. Clinical frailty adds to acute illness severity in predicting mortality in hospitalized older adults: an observational study. Eur J Intern Med. 2016;35:24-34.

15. Kaehr EW, Pape LC, Malmstrom TK, Morley JE. FRAIL-NH predicts outcomes in long term care. J Nutr Health Aging. 2016;20:192-198.

16. Hubbard RE, Peel NM, Samanta M, et al. Derivation of a frailty index from the interRAI acute care instrument. BMC Geriatr. 2015;15:27.

17. Morley JE, Vellas B, van Kan GA, et al. Frailty consensus: a call to action. J Am Med Dir Assoc. 2013;14:392-397.

18. Kelaiditi E, Cesari M, Canevelli M, et al. Cognitive frailty: rational and definition from an (I.A.N.A./I.A.G.G.) international consensus group J Nutr Health Aging. 2013;17:726-734.

19. Vellas B, Cestac P, Morley JE. Implementing frailty into clinical practice: we cannot wait. J Nutr Health Aging. 2012;16:599-600.

20. McMillan GJ, Hubbard RE. Frailty in older inpatients: what physicians need to know. $Q J$ Med. 2012;105:1059-1065.

21. Cesari M, Marzetti E, Thiem U, et al. The geriatric management of frailty as paradigm of "The end of the disease era". Eur J Intern Med. 2016; 31:11-14.

22. Romero-Ortuno R, O'Shea D. Fitness and frailty: opposite ends of a challenging continuum! Will the end of age discrimination make frailty assessments an imperative? Age Ageing. 2013;42:279-280.

23. Muscedere J, Andrew MK, Bagshaw SM, et al. Screening for frailty in Canada's health care system: a time for action. Can J Aging. 2016;35: 281-297.

24. Mitnitski A, Graham AB, Mogilner AJ, Rockwood K. Frailty, fitness and late-life mortality in relation to chronological and biological age BMC Geriatr. 2002;2:1

25. Bouillon K, Kivimaki M, Hamer M, et al. Measures of frailty in population-based studies: an overview. BMC Geriatr. 2013;13:64.

26. Abellan van Kan G, Rolland Y, Bergman H, Morley JE, Kritchevsky SB, Vellas B. The I.A.N.A. Task Force on frailty assessment of older people in clinical practice. J Nutr Health Aging. 2008;12:29-37.

27. Bieniek J, Wilcznski K, Szewieczek J. Fried frailty phenotype assessment components as applied to geriatric inpatients. Clin Interv Aging. 2016;11:453-459.

28. Lisiak M, Uchmanowicz I, Wontor R. Frailty and quality of life in elderly patients with acute coronary syndrome. Clin Interv Aging. 2016;11 553-562.

29. Verloo H, Goulet C, Morin D, von Gunten A. Association between frailty and delirium in older adult patients discharged from hospital. Clin Interv Aging. 2016;11:55-63.

30. Ritt M, Schwarz C, Kronawitter V, et al. Analysis of Rockwood et al's Clinical Frailty Scale and Fried et al's frailty phenotype as predictors of mortality and other clinical outcomes in older patients who were admitted to a geriatric ward. J Nutr Health Aging. 2015;19:1043-1048.
31. Rockwood K, Theou O, Mitnitski A. What are frailty instruments for? Age Ageing. 2015;44:545-547.

32. Howlett SE, Rockwood MR, Mitnitski A, Rockwood K. Standard laboratory tests to identify older adults at increased risk of death. $B M C$ Med. 2014;12:171.

33. Rockwood K, Silvius JL, Fox RA. Comprehensive geriatric assessment: helping your elderly patients maintain functional well-being. Postgrad Med. 1998;103:247-264.

34. Rockwood K, Rockwood MR, Mitnitski A. Physiological redundancy in older adults in relation to the change with age in the slope of a frailty index. J Am Geriatr Soc. 2010;58:318-323.

35. Ritt M, Radi KH, Schwarz C, Bollheimer LC, Sieber CC, Gaßmann KG. A comparison of frailty indexes based on a comprehensive geriatric assessment for the prediction of adverse outcomes. J Nutr Health Aging. 2016;20:760-767.

36. Rockwood K, Andrew M, Mitnitski A. A comparison of two approaches to measuring frailty in elderly people. J Gerontol A Biol Sci Med Sci. 2007;62:738-743.

37. Ritt M, Ritt JI, Sieber CC, Gaßmann KG. Comparing the predictive accuracy of frailty, comorbidity, and disability for mortality: a 1-year follow-up in patients hospitalized in geriatric wards. Clin Interv Aging. 2017;12:293-304

38. Rockwood K, McMillan M, Mitnitski A, Howlett SE. A frailty index based on common laboratory tests in comparison with a clinical frailty index for older adults in long-term care facilities. J Am Med Dir Assoc. 2015; $16: 842-847$

39. Blodgett JM, Theou O, Howlett SE, Wu FC, Rockwood K. A frailty index based on laboratory deficits in community-dwelling men predict their risk of adverse health outcomes. Age Ageing. 2016;45:463-468.

40. Mitnitski A, Collerton J, Martin-Ruiz C, et al. Age-related frailty and its association with biological markers of ageing. BMC Med. 2015; 13:161.

41. Rockwood K, Stadnyk K, MacKnight C, McDowell I, Hebert R, Hogan DB. A brief clinical instrument to classify frailty in elderly people. Lancet. 1999;353:205-206.

42. Hinkle DE, Wiersma W, Jurs SG. Applied Statistics for the Behavioral Sciences. 5th ed. Boston: Houghton Mifflin; 2003.

43. Swets JA. Measuring the accuracy of diagnostic systems. Science. 1988;240:1285-1293.

44. Hanley JA, McNeil BJ. A method of comparing the areas under receiver operating characteristic curves derived from the same cases. Radiology. 1983;148:839-843.

45. Chen C, Sia I, Ma HM, et al. The synergistic effect of functional status and comorbidity burden on mortality: a 16-year survival analysis. PLoS One. 2014;9:e106248.

46. Marengoni A, von Strauss E, Rizzuto D, Winblad B, Fratiglioni L. The impact of chronic multimorbidity and disability on functional decline and survival in elderly persons: a community-based, longitudinal study. J Intern Med. 2009;265:288-295.

47. Landi F, Liperoti R, Russo A, et al. Disability, more than multimorbidity, was predictive of mortality among older persons aged 80 years and older. J Clin Epidemiol. 2010;63:752-759.

48. Zekry D, Valle BH, Graf C, et al. Prospective comparison of 6 comorbidity indices as predictors of 1-year post-hospital discharge institutionalization, readmission, and mortality in elderly individuals. $J \mathrm{Am}$ Med Dir Assoc. 2012;13:272-278.

49. Beloosesky Y, Weiss A, Mansur N. Validity of the Medication-based Disease Burden Index compared with the Charlson Comorbidity Index and the Cumulative Illness Rating Scale for Geriatrics: a cohort study. Drugs Aging. 2011;28:1007-1014.

50. Kobayashi LC, Jackson SE, Lee SE, Wardle J, Steptoe A. The development and validation of an index to predict 10-year mortality risk in longitudinal cohort of older English adults. Age Ageing. Epub 2016 Nov 3

51. Melgaard L, Gorst-Rasmussen A, Rasmussen LH, Lip GY, Larsen RB. Vascular disease and risk stratification for ischemic stroke and all-cause death in heart failure patients without diagnosed atrial fibrillation: a nationwide cohort study. Plos One. 2016;11:e0152269. 
52. Mandelli S, Riva E, Tettamanti M, Detoma P, Giacomin A, Lucca U. Mortality prediction in the oldest old with five different equations to estimate glomerular filtration rate: the health and anemia populationbased study. Plos One. 2015;10:e136039.

53. Holroyd-Leduc JM, Mehta KM, Covinsky KE. Urinary incontinence and its association with death, nursing home admission, and functional decline. J Am Geriatr Soc. 2004;52:712-718.

54. Bootsma AM, Buurman BM, Geerlings SE, de Rooij SE. Urinary incontinence and indwelling urinary catheters in acutely admitted elderly patients: relationship with mortality, institutionalization, and functional decline. J Am Med Dir Assoc. 2013;14:147.e7-147.e12.

55. Koloski NA, Jones M, Wai R, Gill RS, Byles J, Talley NJ. Impact of persistent constipation on health-related quality of life and mortality in older community-dwelling women. Am J Gastroenterol. 2013;108: $1152-1158$

56. Gnjidic D, Hilmer SN, Blyth FM, et al. Polypharmacy cutoff and outcomes: five or more medications were used to identify community-dwelling older men at risk of different adverse outcomes. J Clin Epidemiol. 2012;65:989-995.

57. Perna L, Wahl HW, Mons U, Saum KU, Holleczek B, Brenner H. Cognitive impairment, all-cause and cause-specific mortality among non-demented older adults. Age Ageing. 2015;44:445-451.

58. Sachs GA, Carter R, Holtz LR, et al. Cognitive impairment: an independent predictor of excess mortality: a cohort study. Arch Intern Med. 2011;155:300-308.

59. Idland G, Engedal K, Bergland A. Physical performance and 13.5-year mortality in elderly women. Scand J Public Health. 2013;41:102-108.

60. DeBuyserSL,PetrovicM, Taes YE, ToyeKR, KaufmanJM, GoemaereS. Physical function measurements predict mortality in ambulatory older men. Eur J Clin Invest. 2013;43:379-386.

61. Streicher M, Themessl-Huber M, Schindler KE, Sieber CC, Hiesmayr M, Volkert D. NutritionDay in nursing homes: the association of nutritional intake and nutritional interventions with 6-month mortality in malnourished residents. J Am Med Dir Assoc. 2017;18:162-168.

62. Bahat G, Tufan F, Bahat Z, et al. Observational cohort study on correlates of mortality in older community-dwelling outpatients: the value of functional assessment. Geriatr Gerontol Int. 2015;15:1219-1226.
63. Tabue-Teguo M, Kelaiditi E, Demougeot L, Dartigues JF, Vellas B, Cesari M. Frailty index and mortality in nursing home residents in France: results from the INCUR study. J Am Med Dir Assoc. 2015;16: 603-606.

64. Cabrerizo S, Cuadras D, Gomez-Busto F, Artaza-Artabe I, MarinCiancas F, Malafarina V. Serum albumin and health in older people: review and meta analysis. Maturitas. 2015;81:17-27.

65. Koehler EM, Sanna D, Hansen BE, et al. Serum liver enzymes are associated with all-cause mortality in an elderly population. Liver Int. 2014;34:296-304.

66. Wulaningsih W, Homberg L, Garmo H, et al. Serum lactate dehydrogenase and survival following cancer diagnosis. Br J Cancer. 2015;113: 1389-1396.

67. Liu XH, Meng QH, Ye Y, Hildebrandt MA, Gu J, Wu X. Prognostic significance of pretreatment serum levels of albumin, LDH and total bilirubin in patients with non-metastatic breast cancer. Carcinogenesis. 2015;36:243-248

68. Searle S, Mitnitski A, Gahbauer EA, Gill TM, Rockwood K. A standard procedure for creating a frailty index. BMC Geriatr. 2008;8:24.

69. Rockwood K, Mitnitski A. Limits to deficit accumulation in elderly people. Mech Ageing Dev. 2006;127:494-496.

70. Matzen LE, Jepsen DB, Jesper R, Masud T. Functional level at admission is a predictor of survival in older patients admitted to an acute geriatric unit. BMC Geriatr. 2012;12:32.

71. Avelino-Silva TJ, Farfel JM, Curiati JA, Amaral JR, Campora F, Jacob-Filho W. Comprehensive geriatric assessment predicts mortality and adverse outcomes in hospitalized older adults. BMC Geriatr. 2014; $14: 129$.

72. Theou O, Cann L, Blodgett J, Wallace LM, Brothers TD, Rockwood K. Modifications of the frailty phenotype criteria: systematic review of the current literature and investigation of 262 frailty phenotypes in the survey of health, ageing, and retirement in Europe. Ageing Res Rev. 2015;21:78-94.

73. Mulasso A, Roppolo M, Giannotta F, Rabaglietti E. Associations of frailty and psychosocial factors with autonomy in daily activities: a cross-sectional study in Italian community-dwelling older adults. Clin Interv Aging. 2016;11:37-45.
Clinical Interventions in Aging

\section{Publish your work in this journal}

Clinical Interventions in Aging is an international, peer-reviewed journal focusing on evidence-based reports on the value or lack thereof of treatments intended to prevent or delay the onset of maladaptive correlates of aging in human beings. This journal is indexed on PubMed Central, MedLine,

\section{Dovepress}

CAS, Scopus and the Elsevier Bibliographic databases. The manuscript management system is completely online and includes a very quick and fair peer-review system, which is all easy to use. Visit http://www.dovepress. com/testimonials.php to read real quotes from published authors. 